\title{
MESONS OF ABNORMAL CHARGE PARITY
}

\author{
By \\ L. GÁLFI \\ INSTITUTE FOR THEORETICAL PHYSICS OF THE ROLAND EÖTVÖS UNIVERSITY, BUDAPEST
}

(Presented by K. Nagy. - Received 15. VIII. 1967)

\section{The quark model of the mesons}

The strong interacting particles can be classified by assuming that there exist three fundamental particles, the quarks. The fundamental quantum numbers of the three quarks are:

Table 1

\begin{tabular}{c|c|c|c|c|c|c}
\hline & $Q / \boldsymbol{c}$ & $\begin{array}{c}S \\
\text { strangeness }\end{array}$ & $\boldsymbol{B}$ & $\begin{array}{c}S \\
\text { spin }\end{array}$ & $\begin{array}{c}\boldsymbol{I} \\
\text { isospin }\end{array}$ & \multicolumn{1}{|c}{$I_{3}$} \\
\hline $\boldsymbol{p}$ & $2 / 3$ & 0 & $1 / 3$ & $1 / 2$ & $1 / 2$ & $1 / 2$ \\
$\boldsymbol{n}$ & $-1 / 3$ & 0 & $1 / 3$ & $1 / 2$ & $1 / 2$ & $-1 / 2$ \\
$\lambda$ & $-1 / 3$ & -1 & $1 / 3$ & $1 / 2$ & 0 & 0
\end{tabular}

We can imagine baryons in the simplest way, as the bound-systems of three quarks; mesons, as the bound-systems of one quark and one antiquark. Let us examine, what kind of mesons of quantum numbers $J^{P C}$ can be produced in this way.

a) If $\boldsymbol{P}_{x}$ is the space-, $\boldsymbol{P}_{s}$ is the spin-, $\boldsymbol{P}_{q}$ is the charge-reversal operator, then for every two-fermion system $P_{x} P_{s} P_{q}=-1$.

b) If the resultant orbital momentum of the two particles is $l$, the resultant spin is $S$, then $P_{x}=(-1)^{l}, P_{s}=(-1)^{s+1}$.

c) The parity of the quark - antiquark system equals $P=(-1) \mathbf{I}^{+1}$.

Using these arguments it is easy to see that if $N(>0)$ is even, $(N$ is the spin of the meson), then we can explain the mesons of quantum numbers $J^{P C}=$ $=N^{-+}, N^{++}, N^{--}$, as the bound systems of one quark and one antiquark; if $N$ is odd, then the mesons of $J^{P C}=N^{+-}, N^{--}, N^{++}$; if $N=0$, then the mesons of $J^{P C}=\mathrm{O}^{-+}, \mathrm{O}^{++}$. We shall refer to these mesons as mesons of normal charge parity.

$\mathrm{U}_{\mathrm{p}}$ to now mesons of quantum numbers $J^{P C}=\mathrm{O}^{+-}, \mathrm{O}^{--}, \mathrm{I}^{-+}$have not been observed. We cannot explain these mesons as the bound systems of 
one quark and one antiquark. Let us refer to them as mesons of abnormal charge parity. Now the question can be asked: what kind of properties would they have and how could they be observed. It is well worth examining, whether the fact that mesons of abnormal charge parity have not been discovered is an argument in favour of the quark model, or whether it is only connected with the difficult observation of these particles.

We examine the strong and electromagnetic decays of the mesons of abnormal charge parity of spin 0 and 1 . The decay products are sought among scalar, vector, and tensor mesons. We do not deal with strange particles. Determining the allowed decays, we seek the simplest vertex for the given transition. The effective Hamiltonian, equivalent with the vertex, is composed from the field-functions of the particles - participating in the transition and from the derivatives of fields. Lorentz symmetry, $P$ conservation, isotope invariance and gauge invariance for photons is assumed.

\section{Isoscalar mesons of quantum numbers $J^{P C}=0^{+-}$}

It is assumed that this hypothetical meson $M$ is neutral, its isospin vanishes. If the mass of the $M$ meson increases, new decays become allowed. Fig. 1 shows the thresholds of the communicating channels. (The mass-unit is the mass of $\pi$ mesons).

Let us examine the decay $M \Rightarrow 5 \pi$. The simplest invariant Hamiltonian is:

$$
H(x)=\frac{1}{m^{6}} \varepsilon_{\alpha \beta \gamma \delta} \partial_{\alpha} M(x)\left[\varphi(x) \cdot \partial_{\beta} \varphi(x)\right]\left[\partial_{\gamma} \varphi(x)\left\{\varphi(x) \partial_{\delta} \varphi(x)\right\}\right] .
$$

Here $M(x), \varphi(x)$ are the fields of $M$ meson and $\pi$ meson, -- in isotope space $M(x)$ is a scalar function, $\varphi(x)$ is a vector function $-m$ is a coupling constant of mass dimension, $\varepsilon_{\alpha \beta \gamma \delta \varepsilon}$ is the totally antisymmetric pseudotensor. We calculate one term of the isotope invariant Hamiltonian. The transition probability is

$$
\begin{gathered}
w=\Gamma=\frac{1}{\tau}=\frac{M_{0}}{(2 \pi)^{5} m^{12}} \int \mathrm{d} k_{1} \mathrm{~d} k_{2} \mathrm{~d} k_{4} \mathrm{~d} k_{4} \mathrm{~d} k_{5} \times \\
\times \frac{\left(k_{1} k_{2} k_{3}\right)^{2} \delta^{3}\left(k_{1}+k_{2}+k_{3}+k_{4}+k_{5}\right) \delta\left(M_{0}-\varepsilon_{1}-\varepsilon_{2}-\varepsilon_{3}-\varepsilon_{4}-\varepsilon_{5}\right)}{\sqrt{m_{0}^{2}+k_{1}^{2}} \sqrt{m_{0}^{2}+k_{2}^{2}} \sqrt{m_{0}^{2}+k_{3}^{2}} \sqrt{m_{0}^{2}+k_{4}^{2}} \sqrt{m_{0}^{2}+k_{5}^{2}}} .
\end{gathered}
$$

Here $m_{0}$ and $M_{0}$ are the mass of $\pi$ meson and $M$ meson, $k_{i}$ and $\varepsilon_{i}$ are the momentum and energy of the $i$-th $\pi$ meson, we are in that coordinate-system in which the momentum of the $M$ meson vanishes. We calculate the integral in the following approximation: 
a) $\left(k_{1} k_{2} k_{3}\right)^{2}=\left|k_{1}\right|^{2}\left|k_{2}\right|^{2}\left|k_{3}\right|^{2}$;

b) $k_{i} \Rightarrow \bar{k}$, where $5 \sqrt{m_{0}^{2}+\bar{k}^{2}}=M_{0}$;

c) the domain of integration is determined by $\tilde{k}$;

d) the masses of $\pi^{+}, \pi^{-}$and $\pi^{0}$ are equal.

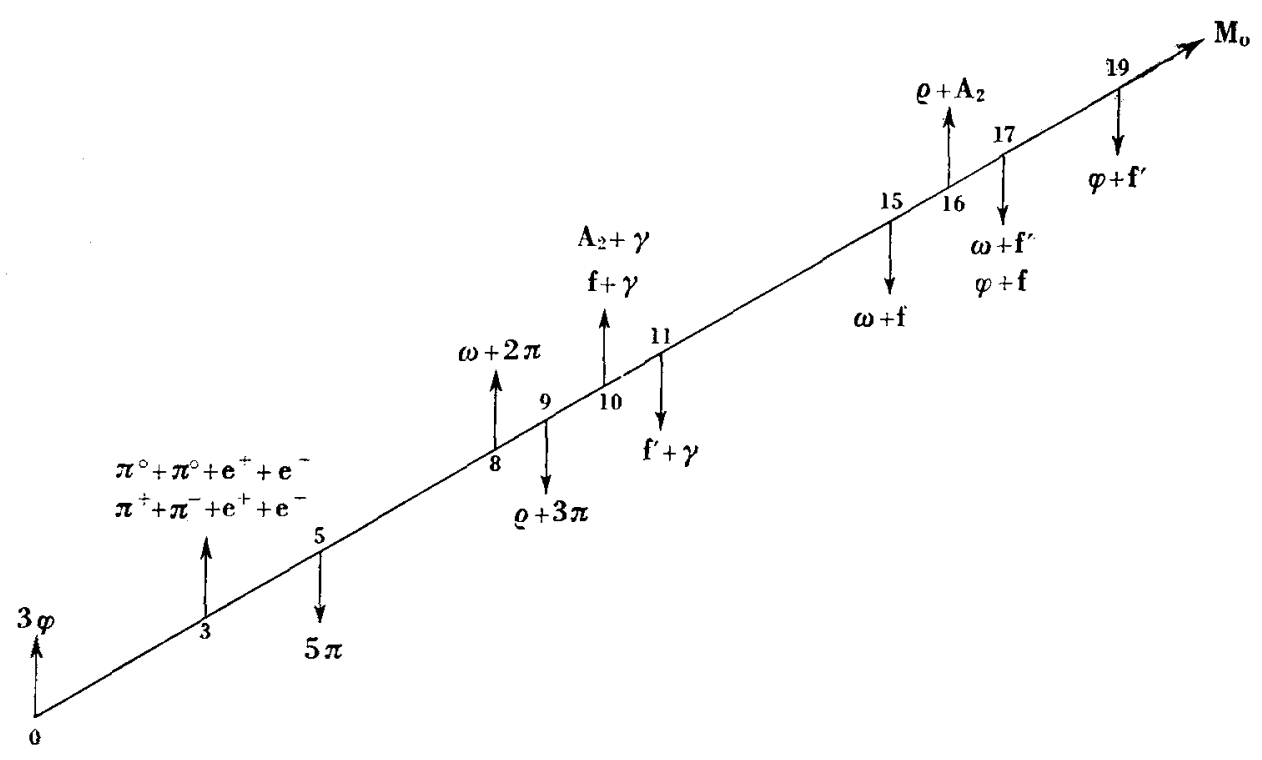

Fig. I

The result is: ( $a$ is defined as $M_{0} / m_{0}, \hbar=c=1$ )

$$
w=\Gamma=\frac{1}{\tau}=\frac{3125 m_{0}^{13}}{(2 \pi)^{5} m^{12}} \frac{\sqrt{\frac{a^{2}}{25}-1}{ }^{17}}{a^{4}}
$$

The numerical results are given by Table 2 .

Table 2

\begin{tabular}{c|c|c|c|c}
\hline \multirow{2}{*}{$a$} & \multicolumn{2}{|c|}{$m=m_{\pi}$} & \multicolumn{2}{|c}{$m=m_{a}$} \\
\cline { 2 - 5 } 6 & $\tau(\mathrm{sec})$ & $\Gamma(\mathrm{MeV})$ & $\tau(\mathrm{sec})$ & $\Gamma(\mathrm{MeV})$ \\
\hline 7 & $5 \cdot 10^{-17}$ & $\sim 0$ & $2 \cdot 10^{-8}$ & $\sim 0$ \\
8 & $5 \cdot 10^{-20}$ & $\sim 0$ & $4 \cdot 10^{-11}$ & $\sim 0$ \\
9 & $10^{-21}$ & 0,6 & $8 \cdot 10^{-13}$ & $\sim 0$ \\
$10^{-22}$ & 6 & $8 \cdot 10^{-14}$ & $\sim 0$
\end{tabular}


The width of the decay $\omega \Rightarrow 3 \pi$ is known to be $\Gamma \sim 10 \mathrm{MeV}$. The calculation was done in this case with the previous approximation. The results are

$$
\Gamma= \begin{cases}727 \mathrm{MeV}, & \text { if } m=m_{\pi}, \\ 0,03 \mathrm{MeV}, & \text { if } m=m_{\varrho}\end{cases}
$$

This means that our results are rather inexact.

A better result can be expected in the strong decays $M \Rightarrow A+B$. Their dynamics is well determined by symmetry requirements, and the integral can be calculated exactly. The coupling constant is dimensionless, it is chosen as 1 . This corresponds better to the coupling constant of strong interactions. The five transitions are mathematically equivalent, the simplest Hamiltonian is

$$
H(x)=M(x) \partial_{\mu} V_{\nu}(x) T_{\mu \nu}(x)
$$

where $V_{n}(x)$ and $T_{\mu v}(x)$ are the fields of the vector, and tensor meson. We sum over the polarisation states of the particles of the final state. The results are given by Table 3 .

Table 3

\begin{tabular}{l|c|c|c|c|c|c}
\hline \multicolumn{1}{c|}{} & $z$ & $\tau(\mathrm{sec})$ & $\Gamma(\mathrm{MeV})$ & $a$ & $\tau(\mathrm{sec})$ & $\Gamma(\mathrm{MeV})$ \\
\hline$M \Rightarrow \omega+f$ & 15 & $3 \cdot 10^{-25}$ & 2300 & 16 & $6 \cdot 10^{-26}$ & $10^{4}$ \\
$M \Rightarrow \omega+f^{\prime}$ & 17 & $3 \cdot 10^{-25}$ & 2300 & 18 & $6 \cdot 10^{-26}$ & $10^{4}$ \\
$M \Rightarrow \Phi+f$ & 17 & $2 \cdot 10^{-25}$ & 3500 & 18 & $4 \cdot 10^{-26}$ & $1,7 \cdot 10^{4}$ \\
$M \Rightarrow \Phi+f^{\prime}$ & 19 & $2 \cdot 10^{-25}$ & 3500 & 20 & $4 \cdot 10^{-26}$ & $1,7 \cdot 10^{4}$ \\
$M \Rightarrow g+A_{5}$ & 16 & $2 \cdot 10^{-25}$ & 3500 & 17 & $5 \cdot 10^{-26}$ & $1,4 \cdot 10^{4}$
\end{tabular}

The electromagnetic decay $M \Rightarrow 3 \gamma$ can be discussed as the decay $\pi^{\circ} \Rightarrow 3 \gamma$ (See [2]). The results are

$$
\tau=\left\{\begin{array}{ll}
5 \cdot 10^{-10} \mathrm{sec}, \text { if } m=m_{\pi} \\
8 \cdot 10^{-3} \mathrm{sec}, \text { if } m=m_{\varrho}
\end{array} \text { and } M_{0}=m_{\pi}\right.
$$

The simplest Hamiltonian for the $M \Rightarrow 2^{+}+\gamma$ transitions is

$$
x(x)=\frac{e}{m} \partial_{\mu} M(x) \partial_{v}, T_{\mu \varrho}(x) F_{\varrho \nu}(x)
$$

The notation is the same as previously, $F \varrho^{1}$ is the electromagnetic field-tensor. The results are given by Table 4 . 
Table 4

\begin{tabular}{l|c|c|r|r|r|r}
\hline \multicolumn{1}{c|}{ decay } & \multicolumn{3}{|c|}{$\tau$ (sec) } & \multicolumn{2}{c}{$\tau$ (sec) } \\
\hline & $a$ & $m=m_{\pi}$ & $m=m_{\varrho}$ & $a$ & $m=m_{\pi}$ & $m=m_{\varrho}$ \\
\hline$M \Rightarrow f+\gamma$ & 10 & $10^{-23}$ & $3 \cdot 10^{-22}$ & 11 & $2 \cdot 10^{-24}$ & $6 \cdot 10^{-23}$ \\
$M \Rightarrow f^{\prime}+\gamma$ & 11 & $2 \cdot 10^{-21}$ & $6 \cdot 10^{-20}$ & 12 & $10^{-23}$ & $3 \cdot 10^{-22}$ \\
$M \Rightarrow A_{2}+\gamma$ & 10 & $10^{-21}$ & $3 \cdot 10^{-20}$ & 11 & $5 \cdot 10^{-24}$ & $2 \cdot 10^{-22}$
\end{tabular}

V. G. Grishin and G. I. Kopylov [1] refer in their paper to the possibility of the $M \Rightarrow \pi^{+}+\pi^{-}+\gamma$ transition, but their Hamiltonian is not gauge-invariant. It can be proved that a gauge-invariant Hamiltonian cannot be constructed. Therefore, we examine the transition $M \Rightarrow \pi^{+}+\pi^{-}+$ $+e^{+}+e^{-}$. This transition is of second order, first the $M$ meson produces a $\pi^{+} \pi^{-}$pair and a virtual photon, the photon produces in the second step an electron-positron pair. The simplest Hamiltonians are

$$
\begin{aligned}
H(x) & =\frac{e}{m} A_{v}(x) \partial_{v}\left(\pi^{+}(x) \pi^{-}(x)\right) M(x), \\
H(y) & =e A_{\mu}(y) \bar{\psi}(y) \gamma_{\mu} \psi(y) .
\end{aligned}
$$

The calculation was made with the same approximation as previously. The results are:

Table 5

\begin{tabular}{c|c|c|c|c|c|c}
\hline life time & $a=3$ & 4 & 5 & 6 & 7 & 8 \\
\hline $\begin{array}{c}m=m_{\pi} \\
\begin{array}{c}\tau(\mathrm{sec}) \\
m=m_{\varrho}\end{array}\end{array}$ & $2 \cdot 10^{-16}$ & $6 \cdot 10^{-16}$ & $6 \cdot 10^{-16}$ & $4 \cdot 10^{-16}$ & $4 \cdot 10^{-16}$ & $4 \cdot 10^{-16}$ \\
& $2 \cdot 10^{-14}$ & $2 \cdot 10^{-14}$ & $10^{-14}$ & $10^{-14}$ & $10^{-14}$
\end{tabular}

3. Isoscalar mesons of quantum numbers $J^{P C}=0^{--}$

Let us name our hypothetical meson G. Fig. 2 shows the thresholds of the communicating channels.

The calculation of the transition probabilities is the same as in 2 . Therefore, we give the results only. In the decay $G \Rightarrow 3 \pi$ the transition probability decreases because of the high centrifugal barrier. In our approximation the decay $G \Rightarrow 5 \pi$ is equivalent with the decay $M \Rightarrow 5 \pi$, its results are given by Table 2 . 
The results of transitions $G \Rightarrow 3 \gamma$ and $G \Rightarrow 2^{+}+\gamma$ are the same as in the case of the $M$ meson. One can construct for the $G \Rightarrow \pi^{+}+\pi^{-}+e^{+}+e^{-}$ transition a complicated Hamiltonian only which contains many derivatives; the transition probability decreases.

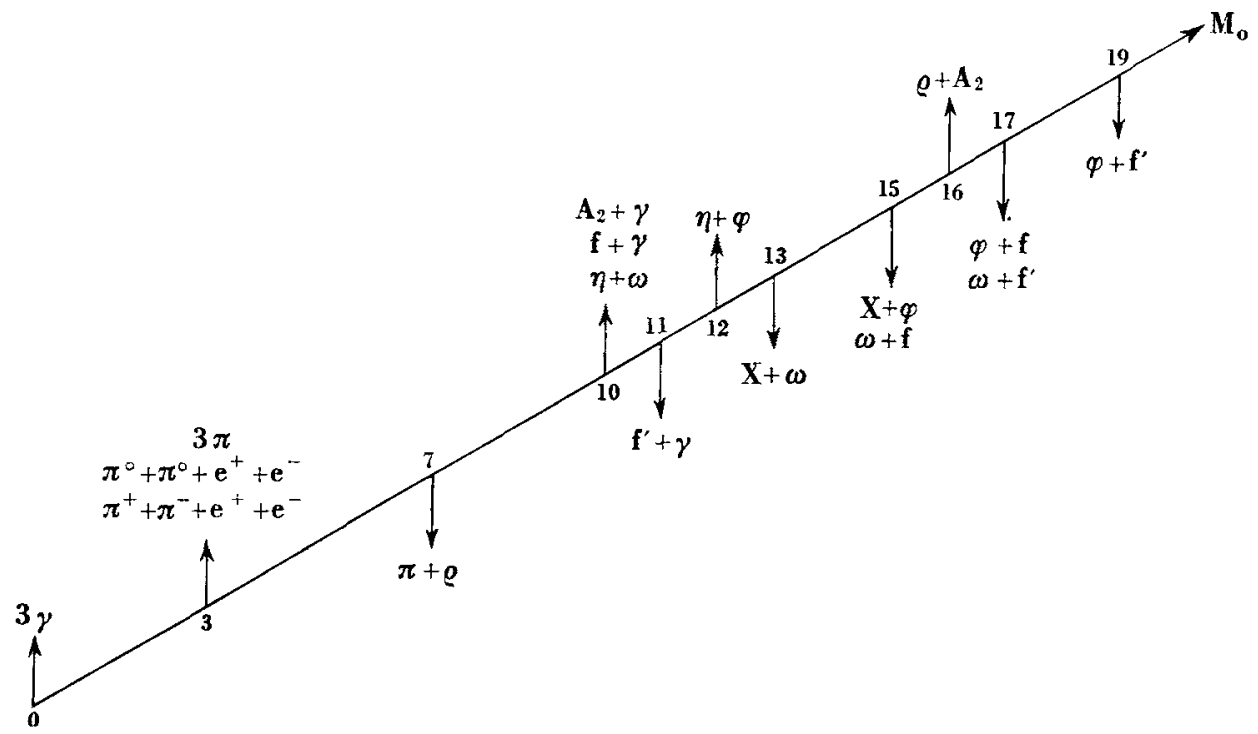

Fig. 2

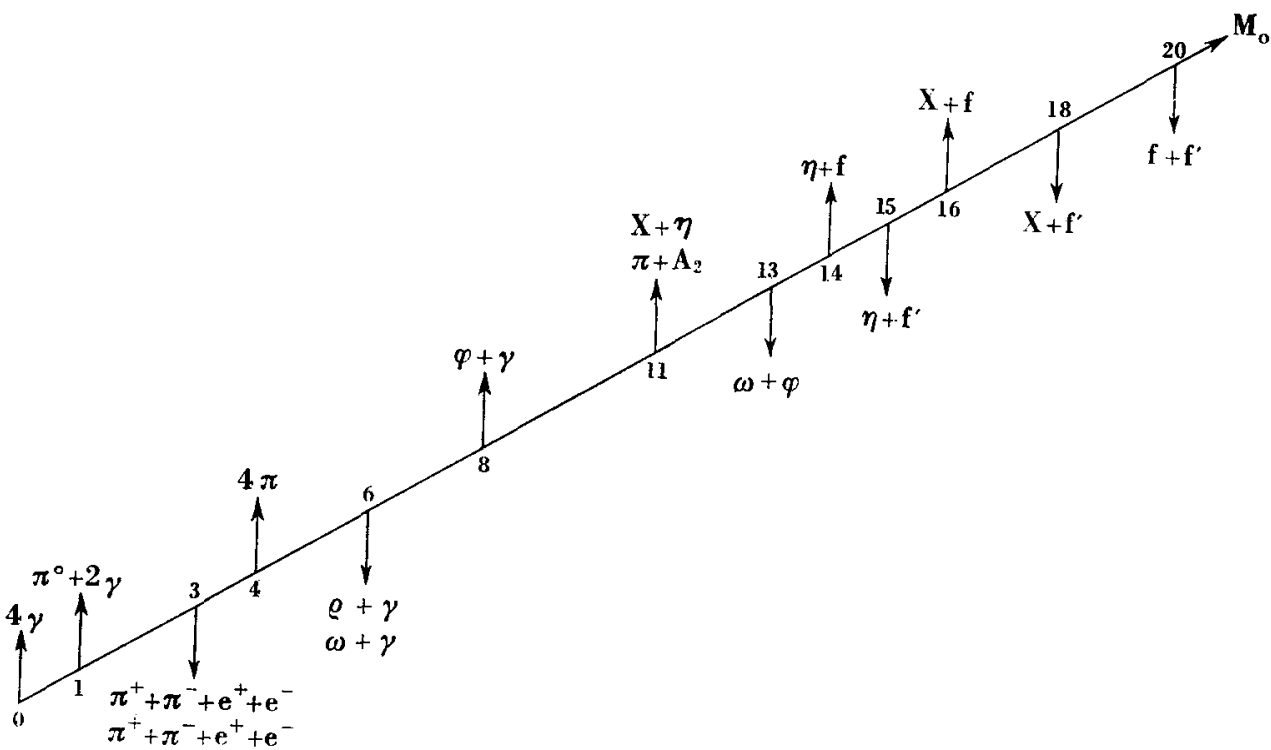

Fig. 3 
Table 6

\begin{tabular}{|c|c|c|c|c|c|c|}
\hline decay & $a$ & $\tau(\mathrm{sec})$ & $\Gamma(\mathrm{MeV})$ & $a$ & $\tau(\mathrm{sec})$ & $\Gamma(\mathrm{MeV})$ \\
\hline$G \Rightarrow \pi+\varrho$ & 7 & $10^{-24}$ & 700 & 8 & $2 \cdot 10^{-25}$ & 3500 \\
\hline$G \Rightarrow X+\omega$ & 13 & $10^{-25}$ & 7000 & 14 & $3 \cdot 10^{-26}$ & $2 \cdot 10^{4}$ \\
\hline$G \Rightarrow X+\Phi$ & 15 & $2 \cdot 10^{-25}$ & 3500 & 16 & $5 \cdot 10^{-26}$ & $1,4 \cdot 10^{4}$ \\
\hline $\boldsymbol{G} \Rightarrow \boldsymbol{u}+\omega$ & 10 & $2 \cdot 10^{-25}$ & 3500 & 11 & $5 \cdot 10^{-26}$ & $1,4 \cdot 10^{4}$ \\
\hline$G \Rightarrow u+\Phi$ & 12 & $4 \cdot 10^{-25}$ & 1750 & 13 & $8 \cdot 10^{-26}$ & $8 \cdot 10^{3}$ \\
\hline
\end{tabular}

Table 7

(Coupling constant is $m=m \varrho$ )

\begin{tabular}{|c|c|c|c|c|c|c|}
\hline decay & $a$ & $\tau(\mathrm{sec})$ & $I^{\prime}(\mathrm{MeV})$ & $a$ & $\tau(\sec )$ & $\Gamma(\mathrm{MeV})$ \\
\hline$G \Rightarrow \omega+f$ & 15 & $8 \cdot 10^{-25}$ & 825 & 16 & $5 \cdot 10^{-26}$ & $1,4 \cdot 10^{4}$ \\
\hline $\boldsymbol{G} \Rightarrow \omega+f^{\prime}$ & 17 & $4 \cdot 10^{-25}$ & 1750 & 18 & $3 \cdot 10^{-26}$ & $2 \cdot 10^{4}$ \\
\hline $\mathbf{G} \Rightarrow \Phi+f$ & 17 & $4 \cdot 10^{-25}$ & 1750 & 18 & $3 \cdot 10^{-26}$ & $\cdot 10^{4}$ \\
\hline $\boldsymbol{G} \Rightarrow \Phi+f^{\prime}$ & 19 & $2 \cdot 10^{-25}$ & 3500 & 20 & $2 \cdot 10^{-26}$ & $3 \cdot 10^{4}$ \\
\hline$G \Rightarrow \varrho+A_{2}$ & 16 & $4 \cdot 10^{-25}$ & 1750 & 17 & $5 \cdot 10^{-26}$ & $1,4 \cdot 10^{4}$ \\
\hline
\end{tabular}

\section{Isoscalar mesons of quantum numbers $J^{P C}=1^{-+}$}

Let us name our third hypothetical meson $L$. Fig. 3 shows the thresholds of the communicating channels. The calculation of the transition probabilities is the same as previously; the results are given by the following Tables.

Table 8

\begin{tabular}{|c|c|c|c|c|}
\hline \multirow{2}{*}{$L \Rightarrow 4 \pi$} & \multicolumn{2}{|c|}{$m=m_{\pi}$} & \multicolumn{2}{|c|}{$m=m_{\varrho}$} \\
\hline & $\tau(\sec )$ & $\Gamma(\mathrm{MeV})$ & $\tau(\mathrm{sec})$ & $I^{r}(\mathrm{MeV})$ \\
\hline$a=5$ & $7 \cdot 10^{-29}$ & $\sim 0$ & $6 \cdot 10^{-16}$ & $\sim 0$ \\
\hline 6 & $3 \cdot 10^{-20}$ & $\sim 0$ & $3 \cdot 10^{-17}$ & $\sim 0$ \\
\hline 7 & $7 \cdot 10^{-21}$ & 0,1 & $6 \cdot 10^{-18}$ & $\sim 0$ \\
\hline 8 & $2 \cdot 10^{-21}$ & 0,3 & $2 \cdot 10^{-18}$ & $\sim 0$ \\
\hline
\end{tabular}

In the decay $L \Rightarrow f+f^{\prime}$ are similar results, the summation over the polarisation states is very complicated. The Hamiltonian for the $L \Rightarrow 4 \gamma$ transition and the calculation are very complicated. The lifetime of the transition $1^{++} \Rightarrow 4 \gamma$ is estimated by Grishin and Kopylov [1]; $\tau \sim 1.23$ sec. 


\section{Table 9}

(Coupling constant is $m=m \varrho$ )

\begin{tabular}{l|c|c|c|c|c|c}
\hline \multicolumn{1}{c|}{ decay } & $a$ & $\tau(\mathrm{sec})$ & $\Gamma(\mathrm{sec})$ & $a$ & $\tau(\mathrm{sec})$ & $\Gamma(\mathrm{MeV})$ \\
\hline$L \Rightarrow X+f^{\prime}$ & 18 & $3 \cdot 10^{-23}$ & 2,3 & 19 & $2 \cdot 10^{-25}$ & 3500 \\
$L \Rightarrow X+f$ & 16 & $3 \cdot 10^{-22}$ & 0,2 & 17 & $2 \cdot 10^{-25}$ & 3500 \\
$L \Rightarrow u+f^{\prime}$ & 15 & $3 \cdot 10^{-22}$ & 0,2 & 16 & $6 \cdot 10^{-25}$ & 1160 \\
$L \Rightarrow u+f$ & 14 & $9 \cdot 10^{-25}$ & 770 & 15 & $10^{-25}$ & 7000 \\
$L \Rightarrow \pi+A_{2}$ & 11 & $10^{-22}$ & 0,6 & 12 & $3 \cdot 10^{-24}$ & 230
\end{tabular}

$$
\begin{aligned}
& L \Rightarrow x+\eta a=11 \tau=9 \cdot 10^{-23} \sec \Gamma= 7 \mathrm{MeV} \\
& 12 \tau=2 \cdot 10^{-24} \sec \Gamma=350 \mathrm{MeV} \\
& L \Rightarrow \omega+\varphi a=13 \tau=6 \cdot 10^{-25} \text { sec } \Gamma=1160 \mathrm{MeV} \\
& 14 \tau=6 \cdot 10^{-26} \text { sec } \Gamma=10^{4} \mathrm{MeV}
\end{aligned}
$$

\begin{tabular}{|c|c|c|c|}
\hline$L=\pi^{\circ}+2 \gamma$ & $a=2$ & 3 & 4 \\
\hline $\begin{array}{r}m=m_{\pi} \\
\tau(\mathrm{sec})\end{array}$ & $6 \cdot 10^{-18}$ & $6 \cdot 10^{-18}$ & $2 \cdot 10^{-19}$ \\
\hline$m=m_{\varrho}$ & $2 \cdot 10^{-16}$ & $2 \cdot 10^{-17}$ & $6 \cdot 10^{-18}$ \\
\hline
\end{tabular}

\begin{tabular}{|c|c|c|c|c|c|c|}
\hline $\begin{aligned} L= & \pi^{+}+\pi^{-}+ \\
& +e^{+}+e^{-}\end{aligned}$ & $a=3$ & 4 & 5 & 6 & 7 & 8 \\
\hline $\begin{array}{l}\quad m=m_{\pi} \\
\tau(\sec )\end{array}$ & $6 \cdot 10^{-16}$ & $4 \cdot 10^{-16}$ & $3 \cdot 10^{-16}$ & $10^{-16}$ & $10^{-16}$ & $10^{-16}$ \\
\hline$m=m_{\varrho}$ & $5 \cdot 10^{-13}$ & $4 \cdot 10^{-13}$ & $3 \cdot 10^{-13}$ & $9 \cdot 10^{-14}$ & $9 \cdot 10^{-14}$ & $9 \cdot 10^{-14}$ \\
\hline
\end{tabular}

Table 10

Table 11

Table 12

\begin{tabular}{c|c|c|c|c|c|c}
\hline \multirow{2}{*}{ decay } & \multicolumn{3}{|c|}{$\tau$ (sec) } & \multicolumn{2}{c}{$r(\mathrm{sec})$} \\
\cline { 2 - 5 } & $a$ & $m=m_{\pi}$ & $m=m_{\ell}$ & $a$ & $m=m_{\pi}$ & $m=m_{\varrho}$ \\
\hline$L \Rightarrow \omega+\gamma$ & 6 & $6 \cdot 10^{-21}$ & $2 \cdot 10^{-19}$ & 7 & $3 \cdot 10^{-22}$ & $10^{-20}$ \\
$L \Rightarrow \Phi+\gamma$ & 8 & $6 \cdot 10^{-21}$ & $2 \cdot 10^{-19}$ & 9 & $6 \cdot 10^{-22}$ & $2 \cdot 10^{-20}$ \\
$L \Rightarrow \varrho+\gamma$ & 6 & $2 \cdot 10^{-20}$ & $6 \cdot 10^{-19}$ & 7 & $6 \cdot 10^{-22}$ & $2 \cdot 10^{-20}$
\end{tabular}




\section{The production of the abnormal mesons}

How could the $M, G$ and $L$ mesons be produced? The direct reversal of the decays examined does not seem suitable. The possibilities are: nucleon as target, charged $\pi$ or $K$ mesons as bombarding particles. If the creating of more than two particles is allowed, then for instance the decay $M \Rightarrow \omega+f$ can be used in the following way: the quantum numbers of $f$ meson can be produced, as the bound state of proton and antiproton; the $\omega$ meson decays into three $\pi$ mesons. Let us write the decay $M \Rightarrow \omega+f$ in the form: $M \Rightarrow$ $\Rightarrow \pi+\pi+\pi+p+\bar{p}$. In this way $M$ meson can be created in the reactions

$$
\pi+p \Rightarrow \pi+\pi+p+M \quad \text { or } \quad p+\bar{p} \Rightarrow \pi+\pi+\pi+M .
$$

It can be proved that the scattering $\pi+N \Rightarrow M+N$ ( $N=$ nucleon) is forbidden because of $C P$-invariance and isotope invariance. It seems that the simplest scattering, in which $M$ meson (similarly $G$ and $L$ meson) can be created is: $K^{-}+p \Rightarrow A+M$. In similar scattering $\pi$ meson is created too: $K^{-}+p \Rightarrow A+\pi^{0}$. The ratio

was estimated;

$$
R \equiv \frac{\sigma\left(K^{-}+p \Rightarrow A+\pi^{0}\right)}{\sigma\left(K^{-}+p \Rightarrow A+M\right)}
$$

$$
R \sim 3 \text { if } M_{M} \equiv 1000 \mathrm{MeV}, K=4000 \frac{\mathrm{MeV}}{C}
$$

( $K$ is the momentum of the $K$ meson), $R \Rightarrow 1$, if $K$ increases. It seems that $M$ mesons are produced with comparable probability to $\pi$ mesons, but the number of $M$ mesons is smaller than the number of $\pi$ mesons.

\section{The discussion of the decays}

What can be said on the basis of the results? If mesons of abnormal charge parity would exist, perhaps their electromagnetic decays could be observed. In these decays few particles are created; the meson, which is created in decay $M \Rightarrow$ meson + photon, decays in general into two or three $\pi$ mesons, and the width is no greater as $10 \mathrm{MeV}$. The widths of the strong decays $(M(G, L) \Rightarrow A+B)$ are so great, that these resonances cannot be observed.

How could one observe for instance the $M$ meson? One must bombard a proton target with $K$ - beam of momentum $4-5 \mathrm{GeV} / c$. The first possibility: three photons are found immediately behind the target, and there is a strong maximum in the distribution of the energy of the photons about $100-200$ $\mathrm{MeV}$. In a bubble chamber one could see the $e^{+}, e^{-}$pairs, produced by the 
photons. The second possibility is the decay $M \Rightarrow \pi^{+}+\pi^{-}+e^{+}+e^{-}$. One must identify the four charged particles, a sharp maximum can be expected in the distribution of energy about $300-1000 \mathrm{MeV}$. The third possibility is the decay $M \Rightarrow 2^{+}$meson $+\gamma$. The mass of the $M$ meson is then about $1000-1800 \mathrm{MeV}$. One could observe the photon on the basis of its pair production, the $2^{+}$meson - for instance $f$ meson - on the basis of its $\pi^{+}-\pi^{-}$production.

Summary: We saw the simplest production of the abnormal mesons. Fig. 4. shows the dominant, observable decay and the expected lifetime of the

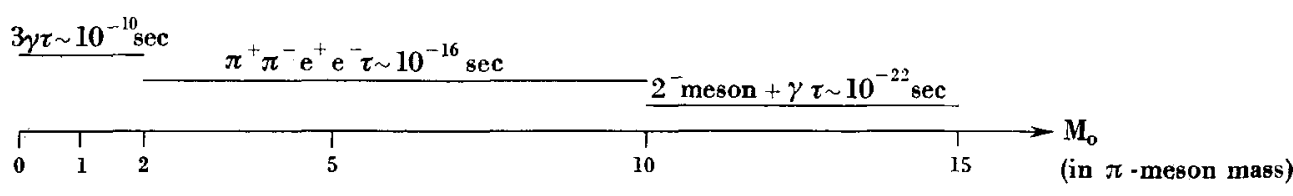

Fig. 4

$M$ meson, as a function of its mass. We think that the strong decays can be observed in a very short mass-interval, theirs widths increase very quickly. One must observe the $G$ und $L$ mesons similarly.

\title{
Acknowledgement
}

I should like to thank Prof. G. Marx, who suggested this problem to me, for his helpful discussions.

\section{REFERENCES}

1. V. G. Grishin and G. I. Kopylov, Il Nouvo Cimento, 37, 962, 1965.

2. L. Gálfi and G. MarX, Acta Phys. Hung., 22, 99, 1966.

3. A. H. Rosenfeld et al., Rev. Mod. Phys. 39, 1, 1967.

\section{МЕЗОНЫ НЕНОРМАЛЬНОЙ ЗАРЯДНОЙ ЧЕТНОСТИ}

\author{
Л. ГАЛФИ \\ P е 3 ю $\mathrm{M} \mathrm{e}$
}

В работе рассматриваются мезоны - в дальнейшем будем их называть мезонами аномальной зарядной четности - которые не представляются связанной системой кварка и антикварка. Исследуются сильные и электромагнитные распады таких мезонов. Выбираются господствующие наблюдаемые виды распадов, оценивается ожидаемое время жизни как функция от массы мезонов, наконец, ищутся наиболее простые способы возбуждения мезонов. Интересно исследовать, что факт необнарудиваемости мезонов аномальной зарядной четности служит ли аргументом модели кварқов, или связан только лишь с трудной наблюдаемостью таких частиц. 